\title{
Alternative Cauchy equation in three unknown functions
}

\author{
Gian Luigi ForTid
}
Dedicated to Professor Ludwig Reich on the occasion of his 80th birthday, with esteem and friendship.

\begin{abstract}
In this paper we deal with the product of two or three Cauchy differences equaled to zero. We show that in the case of two Cauchy differences, the condition of absolute continuity and differentiability of the two functions involved implies that one of them must be linear, i.e., we have a trivial solution. In the case of the product of three Cauchy differences the situation changes drastically: there exists non trivial $\mathcal{C}^{\infty}$ solutions, while in the case of real analytic functions we obtain that at least one of the functions involved must be linear. Some open problems are then presented.
\end{abstract}

Mathematics Subject Classification. Primary 39B22.

Keywords. Alternative Cauchy equation, Analytic solution, $\mathcal{C}^{\infty}$ solution.

\section{Introduction}

We start by considering the following relation

$$
f(x+y)-f(x)-f(y)=\mathfrak{F}(x, y),
$$

where $f: \mathbb{R} \rightarrow \mathbb{R}$ and we assume that $f$ is absolutely continuous and has derivative at each point.

Following [2], the first step consists in giving a suitable representation of both $f$ and $\mathfrak{F}$. Differentiating (1.1) with respect to $x$ we obtain

$$
f^{\prime}(x+y)-f^{\prime}(x)=\frac{\partial \mathfrak{F}(x, y)}{\partial x}
$$

and, setting $x=0$,

$$
f^{\prime}(y)=f^{\prime}(0)+\left.\frac{\partial \mathfrak{F}(x, y)}{\partial x}\right|_{x=0} .
$$


Setting $f^{\prime}(0)=c, c+\left.\frac{\partial \mathfrak{F}(x, y)}{\partial x}\right|_{x=0}=: \phi(y)$ and substituting in (1.2) we obtain

$$
f^{\prime}(x+y)-f^{\prime}(x)=\frac{\partial \mathfrak{F}(x, y)}{\partial x}=\phi(x+y)-\phi(x)
$$

and the following representation of $\mathfrak{F}$ :

$$
\mathfrak{F}(x, y)=\int_{0}^{x}[\phi(t+y)-\phi(t)] d t+k, \quad \phi(0)=0 .
$$

Thus, we conclude that if $f$ and $\mathfrak{F}$ satisfy (1.1), then there exists a continuous function $\phi$ such that (1.5) holds and

$$
f(x+y)-f(x)-f(y)=\int_{0}^{x}[\phi(t+y)-\phi(t)] d t+k .
$$

This simple result will be useful for solving the problem presented in the next section.

\section{Alternative equation in two unknown functions}

In 1978 Marek Kuczma [1] proposed to investigate the following Cauchy alternative functional equation in two unknown functions:

$$
[f(x+y)-f(x)-f(y)][g(x+y)-g(x)-g(y)]=0 ;
$$

obviously there are the so-called trivial solutions, that is when one of the two functions is additive in the domain of our problem. Here we intend to deal with equation (2.1) where $f, g: \mathbb{R} \rightarrow \mathbb{R}$ and $f, g \in \mathcal{C}^{1}$. Equation (2.1) has been extensively studied by L. Paganoni and the present author [3-5] in a more general setting and under weaker regularity conditions and in [4] (Theorem 4)it has been proved that if the pair $(f, g)$ of continuous functions is a solution of (2.1) then one of them is additive, in other words there are no non-trivial continuous solutions of (2.1). This result was obtained through a long procedure and here we will show that the requirement that the two functions are absolutely continuous and differentiable at each point makes the solution of (2.1) rather simple.

From (1.6) in the previous section we have the following:

$$
\begin{aligned}
& f(x+y)-f(x)-f(y)=\mathfrak{F}(x, y)=\int_{0}^{x}[\phi(t+y)-\phi(t)] d t+k_{1}, \\
& g(x+y)-g(x)-g(y)=\mathfrak{G}(x, y)=\int_{0}^{x}[\gamma(t+y)-\gamma(t)] d t+k_{2},
\end{aligned}
$$

where $\phi, \gamma: \mathbb{R} \rightarrow \mathbb{R}$ are two continuous functions, $\phi(0)=\gamma(0)=0$ and $k_{1}, k_{2}$ arbitrary constants; moreover, we have $f(0)=-k_{1}$ and $g(0)=-k_{2}$ and, setting in (2.1) $x=y=0$ we have $f(0) g(0)=k_{1} k_{2}=0$.

Equation (2.1) can be written as

$$
\mathfrak{F}(x, y) \cdot \mathfrak{G}(x, y)=0
$$


and after differentiating with respect to $x$ we have

$$
\mathfrak{G}(x, y)[\phi(x+y)-\phi(x)]+\mathfrak{F}(x, y)[\gamma(x+y)-\gamma(x)]=0
$$

and, with $x=0, k_{2} \phi(y)+k_{1} \gamma(y)=0$. Since we have $k_{1} k_{2}=0$, if $k_{2} \neq 0$, then $k_{1}=0$ and $\phi(y)=0$ for all $y \in \mathbb{R}$; and by (2) this implies $f(x+y)-f(x)-$ $f(y)=0$, i.e., $f(x)=a x, x \in \mathbb{R}$, for some constant $a$. Obviously, if $k_{1} \neq 0$ and $k_{2}=0$, we have $g(x)=b x$.

Now, we assume $k_{1}=k_{2}=0$. Denoting $\Phi_{y}(t):=\phi(t+y)-\phi(t)$ and $\Gamma_{y}(t):=\gamma(t+y)-\gamma(t)$, equation (2.2) becomes

$$
\int_{0}^{x} \Phi_{y}(t) d t \cdot \int_{0}^{x} \Gamma_{y}(t) d t=0, \quad x, y \in \mathbb{R} .
$$

Theorem 2.1. If (2.4) holds for every $x, y \in \mathbb{R}$, then $\Phi_{y}(x) \cdot \Gamma_{y}(x)=0$ for all $x, y \in \mathbb{R}$.

Proof. Let us suppose that the statement is false, then there exist $x_{0}, y_{0} \in \mathbb{R}$ such that $\Phi_{y_{0}}\left(x_{0}\right) \neq 0$ and $\Gamma_{y_{0}}\left(x_{0}\right) \neq 0$. The continuity of $\Phi_{y_{0}}(x)$ and $\Gamma_{y_{0}}(x)$ in $x_{0}$ implies the existence of a positive $\delta$ such that $\Phi_{y_{0}}(x) \neq 0$ and $\Gamma_{y_{0}}(x) \neq 0$ for $\left|x-x_{0}\right|<\delta$. But if

$$
\int_{0}^{x_{0}} \Phi_{y_{0}}(t) d t \neq 0
$$

then

$$
\int_{0}^{x} \Phi_{y_{0}}(t) d t \neq 0, \quad\left|x-x_{0}\right|<\delta,
$$

and, by (2.4), this implies

$$
\int_{0}^{x} \Gamma_{y_{0}}(t) d t=0, \quad\left|x-x_{0}\right|<\delta
$$

and from this we have that $\Gamma_{y_{0}}(x)=0$ for $\left|x-x_{0}\right|<\delta$ : a contradiction.

So, from Theorem 2.1 we have

$$
[\phi(x+y)-\phi(x)][\gamma(x+y)-\gamma(x)]=0, \quad x, y \in \mathbb{R}
$$

and, setting $x=0, \phi(y) \cdot \gamma(y)=0, y \in \mathbb{R}$. Thus, developing (2.8) and considering the last equality, we obtain

$$
\phi(x+y) \gamma(x)+\gamma(x+y) \phi(x)=0, \quad x, y \in \mathbb{R} .
$$

If $\phi\left(x_{0}\right) \neq 0$ for some $x_{0}$, then $\gamma\left(x_{0}\right)=0$ and from (2.9) we have $\gamma\left(x_{0}+y\right)=0$ for all $y \in \mathbb{R}$, hence $\gamma \equiv 0$ and $g(x)=b x$.

Summarizing we have the following

Theorem 2.2. A pair $(f, g)$ of continuously differentiable functions is a solution of (2.1) if and only if at least one of the functions is linear.

Thus, we can say that equation (2.1) has only trivial $\mathcal{C}^{1}$ solutions, i.e., at least one of the factors must be identically zero. 


\section{Alternative equation in three unknown functions}

Consider now the following alternative Cauchy equation in three unknown functions

$[f(x+y)-f(x)-f(y)][g(x+y)-g(x)-g(y)][h(x+y)-h(x)-h(y)] \neq 301)$

In this case we have a completely different situation, i.e., there exist $\mathcal{C}^{\infty}$ functions solving (3.1) and neither of them is additive on the whole $\mathbb{R}$. An example is constructed as follows. Define the following three functions:

$$
\begin{aligned}
& f_{0}(x)=\exp \left\{-\frac{1}{x-1}+\frac{1}{x-2}\right\}+x, \quad 1<x<2, \\
& g_{0}(x)=\exp \left\{-\frac{1}{x-4}\right\}, \quad x>4, \\
& h_{0}(x)=\exp \left\{-\frac{1}{x}+\frac{1}{x-1}\right\}+x, \quad 0<x<1,
\end{aligned}
$$

then we construct these other functions:

$$
\begin{gathered}
f(x)=\left\{\begin{array}{cc}
x, & |x| \leq 1,|x| \geq 2, \\
f_{0}(x), & 1<x<2, \\
-f_{0}(-x), & -2<x<-1 .
\end{array}\right. \\
g(x)=\left\{\begin{array}{cc}
-g_{0}(-x), & x<-4, \\
0, & -4 \leq x \leq 4, \\
g_{0}(x), & x>4 .
\end{array}\right. \\
h(x)=\left\{\begin{array}{cc}
x, & |x| \geq 1, \\
h_{0}(x), & 0<x<1, \\
0, & x=0, \\
-h_{0}(-x), & -1<x<0 .
\end{array}\right.
\end{gathered}
$$

The three functions $f, g$ and $h$ are $\mathcal{C}^{\infty}$ on $\mathbb{R}$ and a simple (and tedious) check shows that the triple $(f, g, h)$ is a solution of (3.1).

Things change completely if we increase the regularity of the functions. We assume that the triple $(f, g, h)$ is a solution of equation (3.1), with all functions in $\mathcal{C}(\mathbb{R})$ and at least one of them, say $f$, real analytic (i.e., it is a real function which is the restriction of an analytic function to the real line ). We intend to prove that if neither $g$ nor $h$ is additive, then $f$ is additive, in other words, equation (3.1) has only trivial solutions.

Theorem 3.1. Assume that the triple $(f, g, h)$ is a solution of equation (3.1) with $g$ and $h$ continuous and not linear, and $f$ real analytic, than $f$ is linear, i.e., equation (3.1) has only trivial solutions.

Proof. Since neither $g$ nor $h$ is additive, Theorem 2.2 in Sect. 2 implies that $\mathfrak{G}\left(x_{0}, y_{0}\right) \cdot \mathfrak{H}\left(x_{0}, y_{0}\right) \neq 0$ for some pair $\left(x_{0}, y_{0}\right) \in \mathbb{R}^{2}$ and, thanks to the continuity of $g$ and $h$, there exists a square $Q=\left(x_{0}-\delta, x_{0}+\delta\right) \times\left(y_{0}-\delta, y_{0}+\delta\right)$ 
such that $\mathfrak{G}(x, y) \cdot \mathfrak{H}(x, y) \neq 0$ for all $(x, y) \in Q$. Hence in $Q$ we must have $\mathfrak{F}(x, y)=0$. We write the expansions of $f$ with center in $x_{0}, y_{0}$ and $x_{0}+y_{0}$, valid for $\left|x-x_{0}\right|<\mu$ and $\left|y-y_{0}\right|<\mu$, for a certain positive $\mu$ :

$$
\begin{aligned}
f(x+y) & =f\left(x_{0}+y_{0}\right)+\sum_{k=1}^{\infty} a_{k}\left(x-x_{0}+y-y_{0}\right)^{k} \\
f(x) & =f\left(x_{0}\right)+\sum_{k=1}^{\infty} a_{k}^{\prime}\left(x-x_{0}\right)^{k} ; \quad f(y)=f\left(y_{0}\right)+\sum_{k=1}^{\infty} a_{k}^{\prime \prime}\left(y-y_{0}\right)^{k}(3.2)
\end{aligned}
$$

Since $\mathfrak{F}\left(x_{0}, y_{0}\right)=0$, from $(3.2)$, setting for brevity $x-x_{0}=t$ and $y-y_{0}=v$, we have

$$
\sum_{k=1}^{\infty}\left[a_{k}(t+v)^{k}-a_{k}^{\prime} t^{k}-a_{k}^{\prime \prime} v^{k}\right]=0 .
$$

We proceed by nullifying the coefficients of the previous power series; those of first degree are $a_{1}-a_{1}^{\prime}$ and $a_{1}-a_{1}^{\prime \prime}$ and by equalling them to 0 we get $a_{1}=a_{1}^{\prime}=a_{1}^{\prime \prime}$. Going to the second degree we obtain

$$
a_{2}-a_{2}^{\prime}=0, \quad a_{2}-a_{2}^{\prime \prime}=0, \quad 2 a_{2}=0,
$$

so $a_{2}=a_{2}^{\prime}=a_{2}^{\prime \prime}=0$. Suppose $a_{n}=a_{n}^{\prime}=a_{n}^{\prime \prime}=0$, then (3.3) becomes

$$
\sum_{k=n+1}^{\infty}\left[a_{k}(t+v)^{k}-a_{k}^{\prime} t^{k}-a_{k}^{\prime \prime} v^{k}\right]=0
$$

and by nullifying the coefficients of terms of degree $n+1$ we get

$$
a_{n+1}-a_{n+1}^{\prime}=0, \quad a_{n+1}-a_{n+1}^{\prime \prime}=0, \quad a_{n+1}=0,
$$

so $a_{n+1}=a_{n+1}^{\prime}=a_{n+1}^{\prime \prime}=0$ and by induction we conclude that $a_{k}=a_{k}^{\prime}=a_{k}^{\prime \prime}=$ 0 for all integers $k$ and $f(x)=f\left(x_{0}\right)+a_{1}\left(x-x_{0}\right)$. Because of the analyticity of $f$ we have that it is affine on the whole $\mathbb{R}$, i.e., $f(x)=\alpha+\beta x$. Since

$0=f\left(x_{0}+y_{0}\right)-f\left(x_{0}\right)-f\left(y_{0}\right)=\alpha+\beta x_{0}+\beta y_{0}-\alpha-\beta x_{0}-\alpha-\beta y_{0}=(-8 x 7)$ we obtain that $f$ is linear.

\section{Further results and open problems}

In this section we present some other results, namely some conditions giving only trivial solutions of equation (3.1) and we prove by giving examples that without them there exist non trivial solutions.

We start by using the simple result given in Sect. 1 and used in Sect. 2 for solving (2.1). Assume that the three functions $f, g, h$ are absolutely continuous and differentiable. We have

$$
\mathfrak{F}(x, y) \mathfrak{G}(x, y) \mathfrak{H}(x, y)=0, \quad x, y \in \mathbb{R},
$$


where

$$
\begin{aligned}
\mathfrak{F}(x, y)=\int_{0}^{x}[\phi(t+y)-\phi(t)] d t+k_{1}=\int_{0}^{x} \Phi_{y}(t) d t+k_{1}, & \phi(0)=0, \\
\mathfrak{G}(x, y)=\int_{0}^{x}[\gamma(t+y)-\gamma(t)] d t+k_{2}=\int_{0}^{x} \Gamma_{y}(t) d t+k_{2}, & \gamma(0)=0, \\
\mathfrak{H}(x, y)=\int_{0}^{x}[\psi(t+y)-\psi(t)] d t+k_{3}=\int_{0}^{x} \Psi_{y}(t) d t+k_{3}, & \psi(0)=0 .
\end{aligned}
$$

By differentiating equation (4.1) with respect to $x$, we obtain

$$
\begin{gathered}
{[\phi(x+y)-\phi(x)] \mathfrak{G}(x, y) \mathfrak{H}(x, y)+\mathfrak{F}(x, y)[\gamma(x+y)-\gamma(x)] \mathfrak{H}(x, y)} \\
+\mathfrak{F}(x, y) \mathfrak{G}(x, y)[\psi(x+y)-\psi(x)]=0
\end{gathered}
$$

and setting $x=0$ we have

$$
\phi(y) k_{2} k_{3}+\gamma(y) k_{1} k_{3}+\psi(y) k_{1} k_{2}=0,
$$

where $k_{1}=-f(0), k_{2}=-g(0)$ and $k_{3}=-h(0)$. By setting $x=y=0$ in equation (4.1), we have $f(0) g(0) h(0)=-k_{1} k_{2} k_{3}=0$.

If one of the numbers $k_{1}, k_{2}, k_{3}$, say $k_{1}$, is zero and $k_{2} k_{3} \neq 0$, then from equation (4.4) we have $\phi(y)=0$ for all $y \in \mathbb{R}$ and this implies $\mathfrak{F}(x, y)=0$ for all $x, y \in \mathbb{R}$, hence $f(x)$ is linear. If also $k_{2} k_{3}=0$, equation (4.4) is identically satisfied.

We differentiate equation (4.3) again with respect to $x$ to obtain

$$
\begin{aligned}
& {\left[\phi^{\prime}(x+y)-\phi^{\prime}(x)\right] \mathfrak{G}(x, y) \mathfrak{H}(x, y)+[\phi(x+y)-\phi(x)][\gamma(x+y)-\gamma(x)] \mathfrak{H}(x, y)+} \\
& {[\phi(x+y)-\phi(x)] \mathfrak{G}(x, y)[\psi(x+y)-\psi(x)]+} \\
& {[\phi(x+y)-\phi(x)][\gamma(x+y)-\gamma(x)] \mathfrak{H}(x, y)+\mathfrak{F}(x, y)\left[\gamma^{\prime}(x+y)-\gamma^{\prime}(x)\right] \mathfrak{H}(x, y)+} \\
& \mathfrak{F}(x, y)[\gamma(x+y)-\gamma(x)][\psi(x+y)-\psi(x)]+ \\
& {[\phi(x+y)-\phi(x)] \mathfrak{G}(x, y)[\psi(x+y)-\psi(x)]+} \\
& \mathfrak{F}(x, y)[\gamma(x+y)-\gamma(x)][\psi(x+y)-\psi(x)]+ \\
& \mathfrak{F}(x, y) \mathfrak{G}(x, y)\left[\psi^{\prime}(x+y)-\psi^{\prime}(x)\right]=0 .
\end{aligned}
$$

Setting $x=0$, we obtain

$$
\begin{aligned}
& {\left[\phi^{\prime}(y)-\phi^{\prime}(0)\right] k_{1} k_{2}-\phi(y) \gamma(y) k_{3}-\phi(y) \psi(y) k_{1}-} \\
& \phi(y) \gamma(y) k_{3}+\left[\gamma^{\prime}(y)-\gamma^{\prime}(0)\right] k_{1} k_{3}-\gamma(y) \psi(y) k_{1}- \\
& \phi(y) \psi(y) k_{2}-\gamma(y) \psi(y) k_{1}+\left[\psi^{\prime}(y)-\psi^{\prime}(0)\right] k_{1} k_{2}=0 .
\end{aligned}
$$

Assume now that $k_{1}=k_{2}=0$, then we have

$$
\phi(y) \gamma(y) k_{3}=0,
$$

so, if $k_{3} \neq 0, \phi(x) \gamma(x)=0$ for all $x \in \mathbb{R}$. Under these conditions, our original equation can be written as

$$
\int_{0}^{x} \Phi_{y}(t) d t \cdot \int_{0}^{x} \Gamma_{y}(t) d t \cdot\left[\int_{0}^{x} \Psi_{y}(t) d t+k_{3}\right]=0 .
$$


Since the last factor for $x=0$ is equal to $k_{3}$, it cannot be always zero; if it is different from zero everywhere, then we come back to the alternative equation in two unknown functions studied in Sect. 2 and we conclude that either $f$ or $g$ is linear. Otherwise, let

$$
\mathcal{O}=\left\{(x, y) \in \mathbb{R}^{2}: \int_{0}^{x} \Psi_{y}(t) d t+k_{3}=\mathfrak{H}(x, y) \neq 0\right\}
$$

clearly $\mathcal{O}$ is an open set, it contains all points of the forms $(x, 0)$ and $(0, y)$ and is symmetric with respect to the line $y=x$. Thus, in $\mathcal{O}$ we must have

$$
\int_{0}^{x} \Phi_{y}(t) d t \cdot \int_{0}^{x} \Gamma_{y}(t) d t=0
$$

By repeating the proof of Theorem 2.1, from equation (4.10) we obtain that

$$
\Phi_{y}(x) \cdot \Gamma_{y}(x)=[\phi(x+y)-\phi(x)][\gamma(x+y)-\gamma(x)]=0, \quad(x, y) \in \mathcal{O} .(4.11)
$$

By multiplying and using equation (4.7) we arrive at the following system:

$$
\left\{\begin{array}{l}
\phi(x) \gamma(x)=0, \quad x \in \mathbb{R} \\
\phi(x+y) \gamma(x)+\phi(x) \gamma(x+y)=0, \quad(x, y) \in \mathcal{O} .
\end{array}\right.
$$

Theorem 4.1. Suppose that $x=0$ is an accumulation point of the set where $\gamma$ is different from 0 , then $\mathfrak{F}(x, y)=0$ for all $x, y \in \mathbb{R}$, i.e., the function $f$ is linear.

Proof. Take $x_{0} \in \mathbb{R}$ and consider the point $\left(0, x_{0}\right) \in \mathcal{O}$; since $\mathcal{O}$ is open, there exists $\delta>0$ such that the open square $Q_{\delta}\left(0, x_{0}\right)=(-\delta, \delta) \times\left(x_{0}-\delta, x_{0}+\delta\right)$ is contained in $\mathcal{O}$. By the hypotheses we can choose $x_{1}$ with $\left(0, x_{1}\right) \in Q_{\delta}\left(0, x_{0}\right)$, $\gamma\left(x_{1}\right) \neq 0$ and $x_{0} \in\left(x_{1}+x_{0}-\delta, x_{1}+x_{0}+\delta\right)$. From the system (4.12) we obtain

$$
\phi\left(x_{1}\right)=0, \quad \phi\left(x_{1}+y\right)=0, \quad y \in\left(x_{0}-\delta, x_{0}+\delta\right)
$$

thus $\phi\left(x_{0}\right)=0$ and this implies $\phi \equiv 0$, so we are done.

The following example shows that without the condition stated in the previous proposition there exist non trivial solutions of equation (4.1) with $\mathcal{C}^{\infty}$ functions and $k_{3} \neq 0$ (Note that the example presented in the previous section has $\left.k_{1}=k_{2}=k_{3}=0\right)$. Let $h$ be the following function

$$
h(x)=\left\{\begin{array}{cl}
x, & |x| \geq 1, \\
h_{0}(x), & -1<x<1
\end{array}\right.
$$

where $h_{0}(0)=-k_{3} \neq 0$ and such that $h \in \mathcal{C}^{\infty}$. The open set $\mathcal{O}$ is contained in the union of the three strips $\mathbb{R} \times(-1,1),(-1,1) \times \mathbb{R}$ and that bounded by the lines $y=1-x$ and $y=-1-x$. Now, we must exhibit two $\mathcal{C}^{\infty}$ functions $\phi$ and $\gamma$ satisfying the system (4.12) and not identically zero. Take $\phi(x)=0$ for $x \leq 5$, $\phi(x)>0$ for $x>5, \gamma(x)=0$ for $x \leq 2$ and $x \geq 3$ and $\gamma(x) \neq 0$ for $2<x<3$ and such that both functions are in $\overline{\mathcal{C}}^{\infty}$. Obviously the first equation of system 
(4.12) is satisfied. The second equation is satisfied for $x \leq 2$ and for $3 \leq x \leq 5$ since both $\phi$ and $\gamma$ have value zero. If $2<x<3$ then $\phi(x)=0$ and $\gamma(x)>0$, hence by the second equation of the system we must have $\phi(x+y)=0$ for $(x, y) \in \mathcal{O}$ : this is true since $x+y<4$. If $x>5$ then $\gamma(x)=0$ and $\phi(x) \neq 0$, hence we must have $\gamma(x+y)=0$ for $(x, y) \in \mathcal{O}$ : this is true since either $-1<x+y<1$ or $x+y>4$. The next step consists in proving that $\mathfrak{F}(x, y)$ and $\mathfrak{G}(x, y)$ are not identically zero and the triple $f, g, h$ is a solution of the equation (4.1). We begin with $\mathfrak{F}(x, y)$ : take $x \leq 5$ and $y=1$, then

$$
\mathfrak{F}(x, 1)=\int_{0}^{x}[\phi(t+1)-\phi(t)] d t=\int_{0}^{x} \phi(t+1) d t=\int_{4}^{x} \phi(t+1) d t \neq 0 .
$$

For $\mathfrak{G}(x, y)$ we take $1<x \leq 2$ and $y=1$, then

$$
\mathfrak{G}(x, 1)=\int_{0}^{x}[\gamma(t+1)-\gamma(t)] d t=\int_{0}^{x} \gamma(t+1) d t=\int_{1}^{x} \gamma(t+1) d t \neq 0 .
$$

To prove that the triple $f, g, h$ is a solution of (4.1) we must prove that for each point $(x, y) \in \mathcal{O}$ either $\mathfrak{F}(x, y)$ or $\mathfrak{G}(x, y)$ is zero. Given the symmetry with respect to the line $y=x$ it is enough to consider the two strips $(-1,1) \times \mathbb{R}$ and that bounded by the lines $y=1-x$ and $y=-1-x$ with $x \geq 0$.

Let $-1<x<1$ and $x+y \leq 5$, then

$$
\mathfrak{F}(x, y)=\int_{0}^{x}[\phi(t+y)-\phi(t)] d t=\int_{0}^{x} \phi(t+y) d t=0 ;
$$

let now $-1<x<1$ and $x+y \geq 3$, then

$$
\mathfrak{G}(x, y)=\int_{0}^{x}[\gamma(t+y)-\gamma(t)] d t=\int_{0}^{x} \gamma(t+y) d t=0
$$

and the first strip is covered. Now we go to the second strip; take $(x, y)$ with $1 \leq x \leq 5$ and $-1<x+y<1$, then

$$
\mathfrak{F}(x, y)=\int_{0}^{x}[\phi(t+y)-\phi(t)] d t=\int_{0}^{x} \phi(t+y) d t=0 .
$$

Take now $x>5$ and $-1<x+y<1$, then $y<-4$, so

$$
\begin{aligned}
\mathfrak{G}(x, y) & =\int_{0}^{x}[\gamma(t+y)-\gamma(t)] d t=\int_{0}^{x} \gamma(t+y) d t \\
& =\int_{0}^{5} \gamma(t+y) d t+\int_{5}^{x} \gamma(t+y) d t=0 .
\end{aligned}
$$

Now, we suppose that $k_{1}=k_{2}=k_{3}=0$; in this case our equation becomes

$$
\int_{0}^{x} \Phi_{y}(t) d t \cdot \int_{0}^{x} \Gamma_{y}(t) d t \cdot \int_{0}^{x} \Psi_{y}(t) d t=0 .
$$


We prove the following analogue of Theorem 2.1.

Theorem 4.2. Equation (4.20) implies that $\Phi_{y}(x) \cdot \Gamma_{y}(x) \cdot \Psi_{y}(x)=0$ for all $x, y \in \mathbb{R}$

Proof. Suppose there exist $x_{0}$ and $y_{0}$ such that $\Phi_{y_{0}}\left(x_{0}\right) \cdot \Gamma_{y_{0}}\left(x_{0}\right) \cdot \Psi_{y_{0}}\left(x_{0}\right) \neq 0$, then by continuity there exists $\delta>0$ such that $\Phi_{y_{0}}(x) \cdot \Gamma_{y_{0}}(x) \cdot \Psi_{y_{0}}(x) \neq 0$ for $\left|x-x_{0}\right|<\delta$. If $\int_{0}^{x_{0}} \Phi_{y_{0}}(t) d t \neq 0$, then for $0<\left|x-x_{0}\right|<\delta$ it is $\int_{0}^{x} \Phi_{y_{0}}(t) d t \neq 0$, so by equation (4.20) we have

$$
\int_{0}^{x} \Gamma_{y_{0}}(t) d t \cdot \int_{0}^{x} \Psi_{y_{0}}(t) d t=0, \quad 0<\left|x-x_{0}\right|<\delta
$$

and, by continuity,

$$
\int_{0}^{x} \Gamma_{y_{0}}(t) d t \cdot \int_{0}^{x} \Psi_{y_{0}}(t) d t=0, \quad\left|x-x_{0}\right|<\delta .
$$

If $\int_{0}^{x_{0}} \Gamma_{y_{0}}(t) d t=0$, then we have $\int_{0}^{x} \Gamma_{y_{0}}(t) d t \neq 0$ for $0<\left|x-x_{0}\right|<\delta$, consequently $\int_{0}^{x} \Psi_{y_{0}}(t) d t=0$ for $0<\left|x-x_{0}\right|<\delta$ and, by continuity, $\int_{0}^{x_{0}} \Psi_{y_{0}}(t) d t=$ 0: a contradiction since $\Psi_{y_{0}}(x) \neq 0$ for $\left|x-x_{0}\right|<\delta$.

Setting $x=0$ in $\Phi_{y}(x) \cdot \Gamma_{y}(x) \cdot \Psi_{y}(x)=0$, we get

$$
\phi(y) \gamma(y) \psi(y)=0, \quad y \in \mathbb{R} .
$$

Thus, we obtain the following system

$$
\left\{\begin{array}{l}
\phi(x) \gamma(x) \psi(x)=0, \quad x \in \mathbb{R} \\
\phi(z) \gamma(z) \psi(x)+\phi(z) \gamma(x) \psi(z)-\phi(z) \gamma(x) \psi(x)+\phi(x) \gamma(z) \psi(z)-(4.24) \\
\phi(x) \gamma(z) \psi(x)-\phi(x) \gamma(x) \psi(z)=0, \quad(x, y) \in \mathbb{R}^{2}
\end{array}\right.
$$

The previous calculations show that a first step on a possible road towards the construction of all $\mathcal{C}^{\infty}$ solutions of equation (3.1) consists in solving the systems (4.12) and (4.24) which are consequences of the original one.

Funding Open access funding provided by Universitá degli Studi di Milano within the CRUI-CARE Agreement.

Open Access. This article is licensed under a Creative Commons Attribution 4.0 International License, which permits use, sharing, adaptation, distribution and reproduction in any medium or format, as long as you give appropriate credit to the original author(s) and the source, provide a link to the Creative Commons licence, and indicate if changes were made. The images or other third party material in this article are included in the article's Creative Commons licence, unless indicated otherwise in a credit line to the material. If material is not included in the article's Creative Commons licence and your intended use is not permitted by statutory regulation or exceeds the permitted use, you will need to obtain permission directly from the copyright holder. To view a copy of this licence, visit http:// creativecommons.org/licenses/by/4.0/.

Publisher's Note Springer Nature remains neutral with regard to jurisdictional claims in published maps and institutional affiliations. 


\title{
References
}

[1] Fenyö, I., Forti, G.L.: On the inhomogeneous Cauchy functional equation. Istituto Matematico F. Enriques, Università degli Studi di Milano, Quaderno 46/S (1980)

[2] Forti, G.L., Paganoni, L.: $\Omega$-additive functions on topological groups. In: Constantin Carathéodory: an International Tribute, 312-330, World Sci. Publishing, Singapore (1990)

[3] Forti, G.L., Paganoni, L.: On an alternative Cauchy equation in two unknown functions. Some classes of solutions. Aequationes Math. 42, 271-295 (1991)

[4] Forti, G.L., Paganoni, L.: Local solutions of an alternative Cauchy equation. Publ. Math. Debrecen 44, 51-65 (1994)

[5] Kuczma, M.: Functional equations on restricted domains. Aequationes Math. 18, 1-34 (1978)

\author{
Gian Luigi Forti \\ Dipartimento di Matematica \\ Università degli Studi di Milano \\ via C. Saldini 50 \\ 20133 Milan \\ Italy \\ e-mail: gianluigi.forti@unimi.it
}

Received: October 30, 2020

Revised: March 1, 2021

Accepted: March 3, 2021 